\title{
IBUPROFEN LOADED ORGANOGEL: DEVELOPMENT AND CHARACTERIZATION
}

\author{
CHANDRA PRABHA UPADHYAY, MEENAKSHI BHARKATIYA*
}

Faculty of Pharmacy, B. N. University, Udaipur, Rajasthan, 313001

*Email: meenakshibharkatiya@gmail.com

Received: 29 Oct 2020, Revised and Accepted: 18 Jan 2021

ABSTRACT

Objective: This study aimed to develop and in vitro characterize an organogel $(O G)$ loaded Ibuprofen.

Methods: Organogel $(O G)$ composed of water, isooctane, sorbitan esters, sorbitan monopalmitate (Span-40), and poly(oxyethylene) sorbitan monostearate (Polysorbate-60) was loaded with Ibuprofen. The partial phase behavior of ibuprofen $O G$ was studied to optimize the formulation composition. $1.0 \% \mathrm{w} / \mathrm{w}$ Ibuprofen loaded $O G$ were characterize for rheological, in vitro release and stability study.

Results: Phase diagram showed an isotropic gel region at low water contents, which converted to emulsion on increasing water quantity. The rheological properties of the $O G$ incorporating $1.0 \% \mathrm{w} / \mathrm{w}$ Ibuprofen shows the presence of two Tg's and elastic behavior of gel, reflects the presence of an entangled network of aqueous tubules. The fractal dimension $d_{\mathrm{f}}$ value of 2.1 and 2.3 was obtained for the two curves (elastic and storage modulus), which is indicative of the formation of the densest gel structure. The diffusional release exponent $(n)$ was found to be $\sim 0.7(0.5<n<1)$ which is indicative of non-Fickian, anomalous diffusion of the drug from the $O G$. The in vitro drug release exhibited release @ 7.04\%/h $0.7 / \mathrm{cm}^{2}$ from the $O G$. Ibuprofen containing $O G$ was stable for $28 \mathrm{~d}$ in terms of chemical potency and gel stiffness at $4{ }^{\circ} \mathrm{C}$ and room temperature $\left(\sim 25{ }^{\circ} \mathrm{C}\right)$.

Conclusion: The study indicates the potential of $O G$ for improved transdermal delivery of Ibuprofen.

Keywords: Microemulsion, Organogel, Phase-behavior, Gel-sol transition temperature, Fractal dimension, Ibuprofen, In vitro release, Pharmacokinetics

(c) 2021 The Authors. Published by Innovare Academic Sciences Pvt Ltd. This is an open access article under the CC BY license (https://creativecommons.org/licenses/by/4.0/) DOI: https://dx.doi.org/10.22159/ijap.2021v13i2.40108. Journal homepage: https://innovareacademics.in/journals/index.php/ijap

\section{INTRODUCTION}

Transdermal drug delivery is an attractive route for the systemic delivery of actives since its first commercialization in 1979 [1]. Topical delivery via the skin is a convenient, painless, and non-invasive approach that enables self-administration for patients [2, 3]. Many drugs have been delivered via the transdermal route and have become successful at clinical setup $[4,5]$. It is well established that the outmost layer of the skin poses a major obstacle in permeating the drug across the skin layer. Subsequently, the drug employed should possess certain physicochemical properties of low molecular weight and sufficient hydrophilic-lipophilic balance to successfully penetrate the skin [6]. Non-steroidal anti-inflammatory (NSAIDs) drugs possess favorable physicochemical properties and have been explored for the transdermal route [1, 7]. Among NSAIDs, Ibuprofen is the most commonly used and most prominently prescribed drug to alleviate moderate pain, reduce swelling, control pyrexia, and treat arthritis at higher doses $[8,9]$. However, like other NSAIDs, Ibuprofen carries a risk of gastric ulcers and sometimes bleeding when taken orally.

Over the years, a variety of formulations have been explored to achieve transdermal delivery of a variety of therapeutic agents such as vesicle-based formulations, nanoparticles, microemulsions, and organogels and as so on [10]. Amongst them, organogels $(O G)$ have attracted a great deal of attention due to their relevance in numerous applications such as transdermal drug delivery, separation science, templates, sensors, etc. They are thermoreversible bicontinuous systems consisting of oily surfactant solutions in which water is micro-emulsified, which on cooling develop a self-assembled interconnected network, mostly fibrillar or tubular, that stop macroscopic flow due to surface tension and capillary forces $[11,12]$. The use of organogels has been proposed for the transdermal delivery of various drugs [13-16]. In this view three major types of $O G$ s comprising gelatine $[17,18]$, lecithin [1921] and sorbitan esters [22, 23] have been studied. The $O G$ formulations of our interest are based on non-ionic sorbitan ester surfactants. These belong to a class of surfactants that form lamellar, toroidal, tubular aggregates, and star-shaped clusters in $O G$ s, which were evaluated using optical microscopy, freeze-fracture electron microscopy, and X-ray diffraction techniques. The drug release characteristics from sorbitan ester OGs are also reported [24].
Previously, Upadhyay et al. have evaluated phase behavior, rheological, and drug release characteristics of sorbitan ester $O G$ to optimize the physical properties of the final product because of the equipment like planetary mixers for semisolid processing impart high shear that affects gelation [23]. In this work, we aim to formulate and characterize sorbitan ester $O G$ consisting of Ibuprofen. First, we report the phase diagram for the $O G$ formation at different ratios of hydrophobic/hydrophilic sorbitan ester surfactants consisting of drugs. Then, we investigated rheological characteristics and fractal dimension. Nonetheless, research on topical bases for the improvement in drug permeation is continued and in this paper, we showed that $O G$ is a better alternative for prolongs the effect of Ibuprofen from conventional ibuprofen preparations.

\section{MATERIALS AND METHODS}

\section{Materials}

Span-40, Polysorbate-60, Isooctane and methanol were purchased from Sigma-Aldrich, India. Ibuprofen was a kind gift from IOL Chemicals and Pharmaceuticals Limited India. Distilled water was used in all experiments.

Partial phase-behavior of Sorbitan Esters/Isooctane/Water System

Phase studies were carried out as per published literature with some modification [23] by adding double distilled water to the mixtures of isooctane and surfactant [Span-40/Polysorbate-60 $(1: 1)]$ in different weight ratios using a magnetic stirrer. After the addition of a water drop to the mixture, the mixture was examined visually for optical clarity and through cross polarizers for the presence/absence of the birefringent liquid crystalline phase. The addition of the water was stopped when the turbidity appeared. The concentrations of surfactant, isooctane, and water were then calculated. The appearance of turbidity was chosen as an endpoint at a particular ratio of surfactant/isooctane. The phase behavior of the systems was mapped on phase diagrams, with the top apex representing the isooctane and the other apices representing the surfactant (Span-40/Polysorbate-60 (1:1)) and water. The transparent, homogeneous, non-birefringent area enclosed within the endpoints was considered as water in oil (w/o) microemulsion. Based on the phase behavior study, OG compositions containing $1 \%$ $(\mathrm{w} / \mathrm{w})$ Ibuprofen were formulated and characterized. 


\section{Gel-sol transition temperature and fractional dimension}

The rheology experiments were performed as per published literature [23] using an AR500 stress-controlled rheometer (TA Instruments, U. K.). The dynamic rheology of organogel was studied by using steel parallel plate geometry of radius $20 \mathrm{~mm}$ and angle $0^{\circ}$ with truncation gap $500 \mu \mathrm{m}$. The truncation gap was kept large to avoid breaking of the structures in the organogel sample. Frequency and strain sweep tests were carried out to specify the viscoelastic range. Sol-gel transition studies were conducted under the temperature ramp. The $O G(0.16 \mathrm{~g})$ was applied on the platform of the rheometer set up at a constant angular frequency $(\omega=6.283 \mathrm{rad}$ sec-1) and the temperature was varied from $15{ }^{\circ} \mathrm{C}$ to $35{ }^{\circ} \mathrm{C}$. The storage modulus (elastic), G', and the loss modulus (viscous), G", were recorded and graphically plotted against temperature. The solgel transition temperature was determined as the minima of the Tan $\delta\left(G^{\prime} / G^{\prime \prime}\right)$ vs temperature curve. For the determination of the fractal dimension, the rheometer was set up at a fixed temperature $\left(20^{\circ} \mathrm{C}\right)$ and $\omega$ was varied from 0.5 to 50 rad sec- 1 . The relaxation exponent $\Delta$ was calculated by fitting the curve to the following power-law or a linear logarithmic equation [25].

$$
G^{\prime}(\omega)=\alpha_{1} \omega^{\Delta} \text { and } G^{\prime \prime}(\omega)=\alpha_{2} \omega^{\Delta \prime}
$$

$\log G^{\prime}(\omega)=\log \alpha_{1}+\Delta^{\prime} \log \omega$ and $\log G^{\prime \prime}(\omega)=\log \alpha_{1}+\Delta^{\prime \prime} \log \omega \ldots \ldots$.

where $\mathrm{G}(\omega)$ is the modulus, $\omega$ is the angular frequency (rad sec-1), $\alpha$ is a constant and $\Delta$ is the relaxation exponent. After the determination of relaxation exponent, a relationship of relaxation exponent with the fractal morphology of the gel network was established as published by Muthkumar [26] in the following equation:

$$
\Delta=\mathrm{d}\left(\mathrm{d}+2-2 \mathrm{~d}_{\mathrm{f}}\right) / 2\left(\mathrm{~d}+2-\mathrm{d}_{\mathrm{f}}\right)
$$

Where $d$ is dimension and $d_{f}$ is the fractal dimension. For gel, $d=3$, therefore the equation could be simplified as:

$$
\mathrm{d}_{\mathrm{f}}=(15-10 \Delta) /(6-2 \Delta)
$$

\section{Drug release through synthetic membrane}

The Ibuprofen containing $O G$ was subjected to an in vitro drug release study through a cellophane membrane. The formulation $(1.0 \mathrm{~g})$ was taken in a pretreated dialysis bag (as per the procedure suggested on the supplier, Sigma-Aldrich) clipped on both sides. The dialysis bag was immersed in $100 \mathrm{ml}$ phosphate-buffered saline (PBS, pH 5.5) in the beaker maintained at $37 \pm 1^{\circ} \mathrm{C}$ on a temperaturecontrolled, magnetic stirrer plate. The contents of the beaker were stirred at $50 \mathrm{rpm}$ using the magnetic bar. The sample from the beaker was withdrawn periodically for $24 \mathrm{~h}$ and replaced immediately with an equal volume of PBS, pH 5.5. The samples were analyzed as explained later. The mass released $(\mathrm{M})$ with time $(\mathrm{t})$ was calculated according to the following power law [27].

$$
\frac{\mathrm{M}_{\mathrm{t}}}{\mathrm{M}_{\infty}}=\mathrm{t}^{\mathrm{n}}
$$

where $M_{\infty}$ is mass released after an infinite time.

\section{Stability}

The $O G$ s containing the drug and respective placebos were stored for 15,30 , and $45 \mathrm{~d}$ at $4{ }^{\circ} \mathrm{C}$ and $25 \pm 5^{\circ} \mathrm{C}$ in collapsible aluminum tubes. The drug content was determined spectrophotometrically after dissolving the formulation in methanol against similarly treated placebos. Viscosities of $O G$ formulations were also determined at weekly intervals using a Brookfield Digital Viscometer model LVDVI+(Brookfield Engineering Labs. Inc., USA). The formulations were also subjected to mechanical stress by centrifuging (Remi Centrifuge, India) at $10000 \mathrm{rpm}$ for $20 \mathrm{~min}$ and the volume of phase separation of $O G$ was noted.

\section{RESULTS AND DISCUSSION}

\section{Phase behavior of $O G S$}

Phase behavior of $O G s$ system consisting of isooctane, water, and surfactant mixture (Span-40 and Polysorbate-60) was investigated by plotting the pseudo-ternary phase diagrams in which each corner of the diagram represents $100 \%$ of that particular component. Fig. 1 shows the phase diagram of the isooctane, water, and surfactant mixture. From the phase diagrams, it is clear that the area of $O G s$ region decreased with the addition of the drug (grey). This correlates well with a previous study [23].

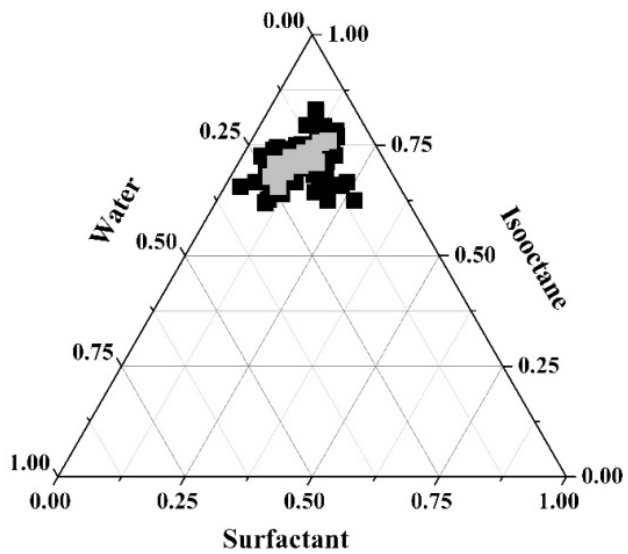

Fig. 1: Triangular phase diagram of $O G$ formulations containing surfactant (Span40/Polysorbate-60, 1:1), isooctane and water. The encapsulated gray area in dark area (without Ibuprofen) shows the phase behavior of ibuprofen containing $O G$

\section{Rheological characteristics of $O G s$}

Gel-sol transition temperature (Tg) of $O G$ formulations was determined using a Rheometer set up at a constant angular frequency $\omega=6.283 \mathrm{rad} \mathrm{sec}^{-1}$ and the temperature, $\mathrm{T}$, were varied from $15{ }^{\circ} \mathrm{C}$ to $35^{\circ} \mathrm{C}$. A representative loss of storage modulus ( $\mathrm{G}^{\prime}$ ) and loss modulus (G") versus temperature curve obtained during the gelation process was plotted as shown in fig. 2. The curves present three domains: high and nearly constant values of $G^{\prime \prime}$ corresponding to sol state, a sharp increase corresponding to gel microstructure formation and the last domain with a tendency to a plateau that could be linked to gel formation. Both the G' and G" values were slightly increased with an increase in drug concentration at a particular temperature. The Tan $\delta$ was calculated as G" $/ G^{\prime}$. The curve between Tan $\delta$ and T was plotted and the Tg was obtained from the minima of the curve (fig. 2 , inset). The parameter $\delta$ is the phase angle or phase shift between the deformation and response that is measured. It is the point where the viscoelasticity of the gel changes abruptly. A careful observation into this curve shows the presence of a shoulder (indicated by an arrow), indicating that the presence of two Tg's. Thus, gel-sol conversion can be explained by a change in the arrangement of the gel network in two stages due to the incorporation of the drug, which suggests that the type of drug encapsulated in organogel affects $O G$ network

The viscoelastic nature of gels is a function of the microstructure network, which can be evaluated using the rheometer set up at fixed temperature $\left(20^{\circ} \mathrm{C}\right)$ and varying $\omega$ from 0.5 to $100 \mathrm{rad} \mathrm{sec}^{-1}$. The elastic and viscous modulus shows an asymptotic increase in values, with the elastic modulus component $\left[G^{\prime}(\omega)\right]$ always having a higher value than the viscous modulus component $\left[G^{\prime \prime}(\omega)\right]$, suggesting the predominantly elastic nature of the sorbitan ester $O G$ within the indicated frequency range. The elastic behavior, in this case, reflects the presence of an entangled network of aqueous tubules. Fractal morphology of $O G$ could be determined from the fractal dimension $\left(d_{\mathrm{f}}\right)$ through a theoretical model for polyelectrolyte gels [25]. The relaxation exponent, $\Delta$, was calculated fitting the power law equation to the $G^{\prime}(\omega), G^{\prime \prime}(\omega)$ vs $\omega$ curves or by plotting logarithm $\left[G^{\prime}(\omega), G^{\prime \prime}(\omega)\right]$ vs logarithm $[\omega]$ curves and determining the slope from the linear fit equation (fig. 3). The $d_{\mathrm{f}}$ value of 2.1 and 2.3 was obtained for the two curves (elastic and storage modulus), which is indicative of the formation of the densest gel structure. The values corroborate with reported studies [23]. 


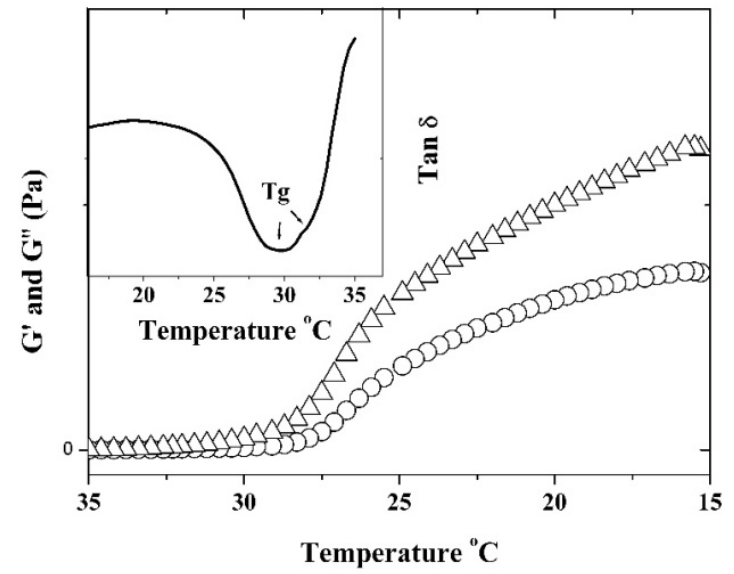

Fig. 2: The temperature-dependent loss of elastic modulus, $G^{\prime}$ (solid legends), and viscous modulus, G" (open legends), of $O G$ formulation, the temperature dependence of $G^{\prime \prime} / G^{\prime} \approx \tan \delta$ curve (inset)

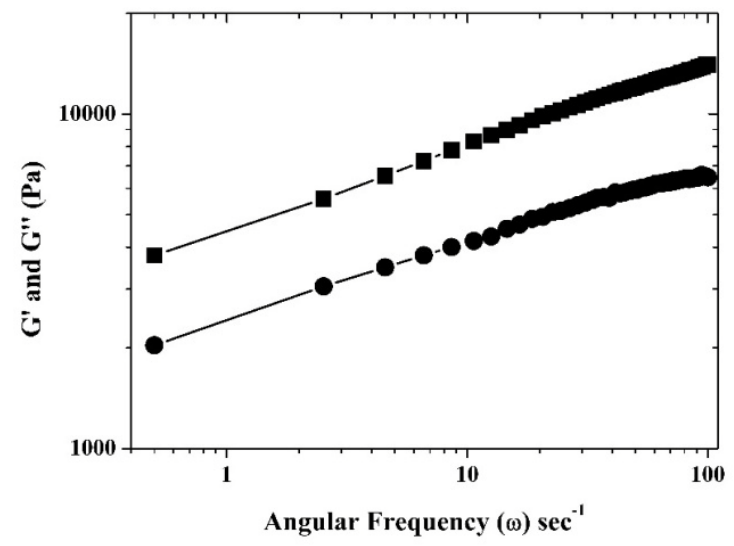

Fig. 3: A representative frequency $(\omega)$ dependence of $G^{\prime}(\omega)$ (circles) and G" $(\omega)$ (triangles) curves of ibuprofen containing $O G$ formulation in logarithmic mode. Relaxation exponents obtained from the slopes of the $\log G^{\prime}(\omega)$ and $\log G^{\prime \prime}(\omega) v s \log \omega$ curves

\section{Drug release from $O G s$}

The kinetics of ibuprofen release from $O G$ was established by plotting a graph between percent cumulative drug release against time and the value of the diffusional release exponent $(n)$ was calculated by fitting a power law to the curve (fig. 4). The value of $n$ was found to be $\sim 0.7(0.5<n<1)$, which is indicative of non-Fickian anomalous diffusion of the drug from the $O G$ [27]. A graph of the percent cumulative amount of drug vs time 0.7 gives the release rate from its slope. The in vitro drug release exhibited release @ 7.04\%/h $0.7 / \mathrm{cm}^{2}$ from the $O G$

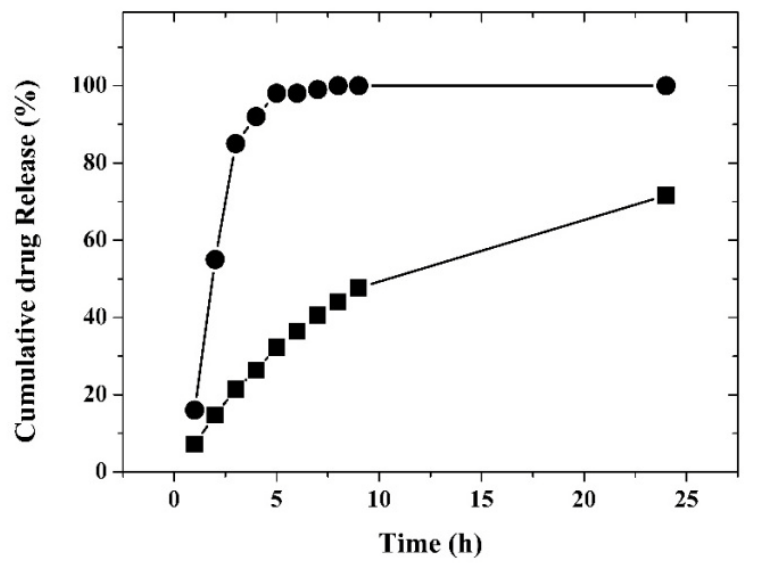

Fig. 4: Cumulative percent drug released versus time curve. The drug release was studies in PBS buffer (pH 5.5) using a cellophane membrane. Solid circles: $O G$ formulation. Solid squares: drug solution

\section{Stability}

The results of stability studies on ibuprofen containing $O G$ formulation are summarized in table 1 . The assay of drugs was more than $98 \%$ in all the formulations at the end of $45 \mathrm{~d}$ at $4{ }^{\circ} \mathrm{C}$ and room temperature $\left(\sim 25^{\circ} \mathrm{C}\right)$ indicating that the chemical stability of ibuprofen in $O G$ formulations was good. The formulations became stiffer over a $45 \mathrm{~d}$ period which was shown by an increase in the viscosity of the $O G$ formulations. The $O G$ formulations were resistant to centrifugal stress.

Table 1: Stability of ibuprofen $O G$ formulation

\begin{tabular}{llll}
\hline S. No. & Formulation code & Time & 30d \\
\cline { 3 - 4 } & & $\mathbf{1 5 d}$ & $98.4 \pm 1.4$ \\
\hline 1. & Assay (\%) & $99.1 \pm 1.2$ & 1600 \\
2. & Viscosity (cps) & 1200 & nil \\
3. & Centrifugal Stress* & nil & $98.7 \pm 0.9$ \\
\hline
\end{tabular}

$\mathrm{n}=3$ (Mean of three determination), *Phase separation on the centrifugation at $1000 \mathrm{rpm}$ for $20 \mathrm{~min}$

\section{CONCLUSION}

Organogel was made using of sorbitan monopalmitate/ polysorbate, isooctane and water. The sol-gel transition with decreasing temperature shows an abrupt denser network formation indicated by the higher fractal dimension value. The drug permeation was slow and displayed anomalous release behavior. The storage stability of $O G$ was good for $45 \mathrm{~d}$. This study proves that sorbitan monopalmitate/polysorbate-based organogel can be a good drug delivery system for transdermal delivery.

\section{ACKNOWLEDGEMENT}

The author wishes to grant the support enhanced by Dean, Faculty of Pharmacy, B. N. University, Udaipur.

\section{AUTHORS CONTRIBUTIONS}

All the authors have contributed equally.

\section{CONFLICT OF INTERESTS}

Declared none

\section{REFERENCES}

1. Tombs EL, Nikolaou V, Nurumbetov G. Transdermal delivery of ibuprofen utilizing a novel solvent-free pressure-sensitive adhesive (PSA): TEPI ${ }^{\circledR}$ technology. J Pharm Innov 2018;13:48-57.

2. Jayprakash R, Hameed J, Anupriya. An overview of the transdermal delivery system. Asian J Pharm Clin Res 2017;10:36-40. 
3. Abualhasan M, Assali M, Jaradat N. Synthesis and formulation of ibuprofen pro-drugs for enhanced transdermal absorption. Int J Pharm Pharm Sci 2015;7:352-4.

4. Akhtar N, Singh V, Yusuf M. Non-invasive drug delivery technology: development and current status of transdermal drug delivery devices, techniques and biomedical applications. Biomedical Engineering/Biomedizinische Technik 2020; 65:243.

5. Watkinson AC, Kearney MC, Quinn HL. Future of the transdermal drug delivery market-have we barely touched the surface? Expert Opin Drug Delivery 2016;13:523-32.

6. Aziz ZAA, Nasir HM, Ahmad A. Enrichment of eucalyptus oil nanoemulsion by micellar nanotechnology: transdermal analgesic activity using hot plate test in rats' assay. Sci Rep 2019;9:13678.

7. Kumar L, Verma S, Singh M. Advanced drug delivery systems for transdermal delivery of non-steroidal anti-inflammatory drugs: a review. Curr Drug Delivery 2018;15:1087-99.

8. Ngo VTH, TB Ibuprofen. In: StatPearls [Internet]. Treasure Island (FL): StatPearls Publishing; 2020.

9. Bushra R, Aslam N. An overview of clinical pharmacology of Ibuprofen. Oman Med J 2010;25:155-66.

10. Zhou X, Hao Y, Yuan L. Nano-formulations for transdermal drug delivery: a review. Chinese Chem Lett 2018;29:1713-24.

11. Esposito CL, Kirilov P, Roullin VG. Organogels, promising drug delivery systems: an update of state-of-the-art and recent applications. J Controlled Release 2018;271:1-20.

12. Fayez SM, Gad S. Formulation and evaluation of etodolac lecithin organogel transdermal delivery systems. Int J Pharm Pharm Sci 2005; 7:325-34.

13. Vigato AA, Querobino SM, de Faria NC. Physico-chemical characterization and biopharmaceutical evaluation of lipidpoloxamer-based organogels for curcumin skin delivery. Front Pharmacol 2019;10:1006.

14. Sharma G, Devi N, Thakur K. Lanolin-based organogel of salicylic acid: evidences of better dermatokinetic profile in imiquimod-induced keratolytic therapy in BALB/c mice model. Drug Delivery Transl Res 2018;8:398-413.
15. Vigato AA, Querobino SM, de Faria NC. Synthesis and characterization of nanostructured lipid-poloxamer organogels for enhanced skin local anesthesia. Eur J Pharm Sci 2019;128:270-8.

16. Vintiloiu A, Leroux JC. Organogels and their use in drug delivery-a review. J Controlled Release 2008;125:179-92.

17. Alfieri ML, Pilotta G, Panzella L. Gelatin-based hydrogels for the controlled release of 5,6-dihydroxyindole-2-carboxylic acid, a melanin-related metabolite with potent antioxidant activity. Antioxidants (Basel) 2020;9:245.

18. Sagiri SS, Singh VK, Kulanthaivel S. Stearate organogel-gelatin hydrogel-based bigels: physicochemical, thermal, mechanical characterizations and in vitro drug delivery applications. J Mech Behav Biomed Mater 2015;43:1-17.

19. Murashova NM, Yurtov EV. Lecithin organogels as prospective functional nanomaterial. Nanotechnol Russ 2015;10:511-22.

20. Chang CE, Hsieh CM, Chen LC. Novel application of pluronic lecithin organogels (PLOs) for local delivery of synergistic combination of docetaxel and cisplatin to improve therapeutic efficacy against ovarian cancer. Drug Delivery 2018;25:632-43.

21. Alsaab H, Bonam S, Bahl D. Organogels in drug delivery: a special emphasis on pluronic lecithin organogels. J Pharm Pharm Sci 2016;19:252-73.

22. Rajpoot K. Acyclovir-loaded sorbitan esters-based organogel: development and rheological characterization. Artif Cells Nanomed Biotechnol 2016;45:1-9.

23. Upadhyay KK, Tiwari C, Khopade AJ. Sorbitan ester organogels for transdermal delivery of sumatriptan. Drug Dev Ind Pharm 2007;33:617-25.

24. Pisal S, Shelke V, Mahadik K. Effect of organogel components on in vitro nasal delivery of propranolol hydrochloride. AAPS PharmSciTech 2004;5:e63.

25. Mohanty B, Bohidar HB. Microscopic structure of gelatin coacervates. Int J Biol Macromol 2005;36:39-46.

26. Muthukumar M. Screening effect on viscoelasticity near the gel point. Macromolecules 1989;22:4656-8.

27. Peppas NA, Brannon-Peppas L. Water diffusion and sorption in amorphous macromolecular systems and foods. J Food Eng 1994;22:189-210. 